\title{
The manufacturing, assembly and acceptance testing of the breadboard Cryogenic Optical Delay Line for DARWIN
}

\author{
T. C. van den Dool*a , F. Kamphues ${ }^{\mathrm{a}}$, W. Gielesen ${ }^{\mathrm{a}}$, M. Dorrepaal ${ }^{\mathrm{a}}$, N. Doelman ${ }^{\mathrm{a}}$,

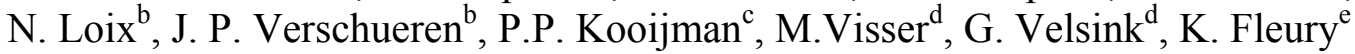

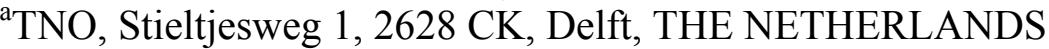 \\ ${ }^{b}$ Micromega-Dynamics, Rue des Chasseurs Ardennais, B-4031, Angleur (Liège), BELGIUM \\ 'SRON, Sorbonnelaan 2, 3584 CA, Utrecht, THE NETHERLANDS \\ ${ }^{\mathrm{d}}$ Dutch Space, Newtonweg 1, 2333 CP, Leiden, THE NETHERLANDS \\ ${ }^{\mathrm{e}} \mathrm{CSL}$, Parc Scientifique du Sart Tilman, Angleur (Liège), BELGIUM
}

\begin{abstract}
TNO, in cooperation with Micromega-Dynamics, SRON, Dutch Space and CSL, has developed a compact breadboard cryogenic Optical Delay Line for use in future space interferometry missions. The work is performed under ESA contract in preparation for the DARWIN mission. The breadboard delay line is representative of a future flight mechanism, with all used materials and processes being flight representative. The delay line has a single stage voice coil actuator for Optical Path Difference (OPD) control, driving a two-mirror cat's eye. Magnetic bearings are used for guiding. They provide frictionless and wear free operation with zero-hysteresis. The manufacturing, assembly and acceptance testing have been completed and are reported in this paper. The verification program, including functional testing at $40 \mathrm{~K}$, will start in the final quarter of 2005.
\end{abstract}

Keywords: optical delay line, ODL, cryogenic, DARWIN, active magnetic bearings, nanopositioning, aperture synthesis

\section{INTRODUCTION}

This paper describes the manufacturing, assembly and acceptance testing of the DARWIN breadboard cryogenic Optical Delay Line (ODL). The delay line is built under ESA contract in preparation for the DARWIN mission [1]. The verification program, including functional testing at $40 \mathrm{~K}$, will start in the final quarter of 2005 . The delay line is shown in figure 1.

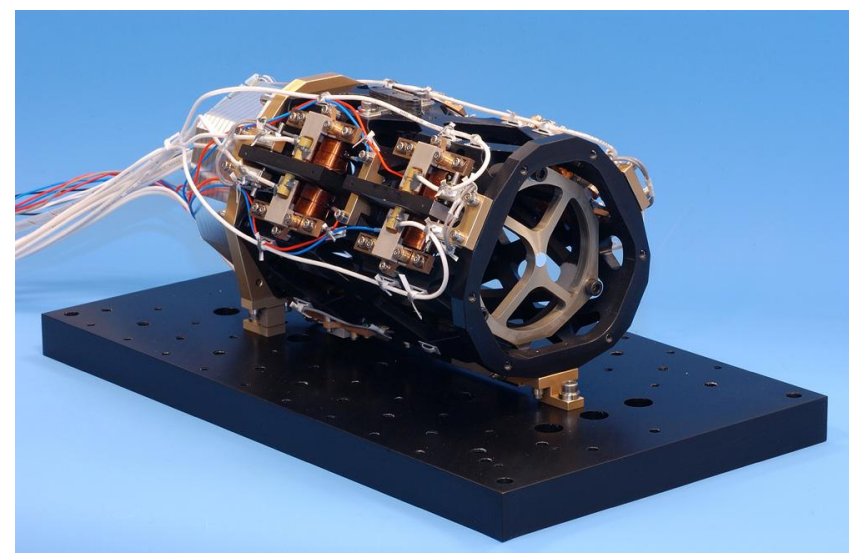

Figure 1 - DARWIN BB Optical Delay Line

- $\quad$ teun.vandendool@tno.nl; phone 31-15-26 92 457; fax 31-15-26 92 111; www.tno.nl

Cryogenic Optical Systems and Instruments XI, edited by James B. Heaney, Lawrence G. Burriesci, Proceedings of SPIE Vol. 5904 (SPIE, Bellingham, WA, 2005) · 0277-786X/05/\$15 · doi: 10.1117/12.616915 
Within this programme, the responsibilities for the design and manufacturing are divided as follows:

- $\quad$ TNO - Project management, systems engineering, optical design, mirror production and OPD control

- Micromega-Dynamics - Magnetic Bearing design and manufacturing

- $\quad$ SRON - OPD Actuator design and manufacturing and cryogenic consultancy

- Centre Spatiale de Liege (CSL) - Coating engineering and 40K TV facility

- Dutch Space - System modelling and 120K TV test support

- Alcatel - DARWIN mission level consultancy

The design and manufacturing phase will be followed by a comprehensive verification program, including functional testing at $40 \mathrm{~K}$.

\section{DESIGN DESCRIPTION}

The DARWIN Optical Delay Line will play an important role in ESA's DARWIN Infrared Nulling Interferometer. The delay line has to equalise the optical path between the free flying telescopes to less than 1 nanometer [2]. The delay lines will also be used for fringe scanning, after course acquisition of the telescope constellation. The delay lines will be placed on the optical bench in the Hub spacecraft and will operate at $40 \mathrm{~K}$ [2]. The dimension of the DARWIN delay line is $120 \times 120 \times 250 \mathrm{~mm}$.

The delay line has a single stage voice coil actuator for Optical Path Difference (OPD) control, driving a two-mirror cat's eye. Magnetic bearings constrain the other five degrees of freedom and provide a frictionless and wear free guiding system. The magnetic bearings use the same controller as the OPD control, eliminating the need for an additional control board. The smooth operation of the magnetic bearings allow single stage OPD control, which contributes to a better optical quality of the cats eye and reduced OPD control complexity. The entire mechanism is constructed from Aluminium 6061, with the exception of a few small components. All parts are constructed from the same material lot, to ensure highly isotropic behaviour. This makes the design fully athermal. Where components with a different CTE are employed, flexures are used to minimise thermal distortions.

The mirrors are plated with a 200 micron layer of Alumiplate ${ }^{\circledR}$, a pure Aluminium that prevents bimetallic effects which can cause differential thermal bending of the mirrors.

More information on the design of the delay line is given in [3].

\section{MIRROR MANUFACTURING}

The cats eye has one parabolic M1 mirror and one flat M2 mirror. Two sets of M1 and M2 mirrors were manufactured. One set for the dynamic delay line and one set for the static delay line (the ESA program comprises two delay lines).

The mirrors were premachined and heat treated by Mecon in Doetinchem, The Netherlands. After heat treatment, the mirrors were plated with pure Aluminium by Alumiplate in Minneapolis, USA. Plating thickness, adhesion and porosity were checked on witness samples. The mirrors were subsequently subjected to cryo stabilisation and diamond turned to its final shape at TNO's optical facility.

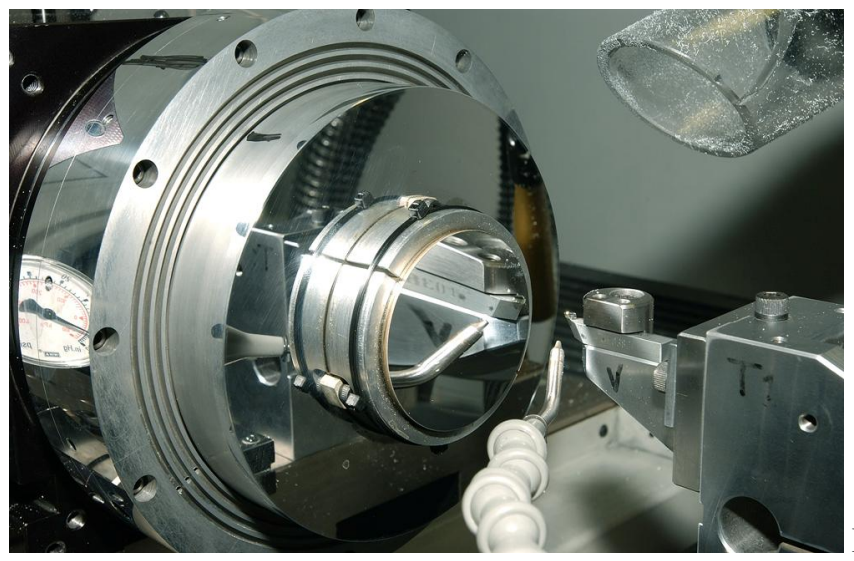

Figure 2 - Diamond turning of M1 mirror 
The WFE of all mirrors was measured with a WYKO interferometer. The M1 is mounted in front of the WYKO. The interferometer beam is focussed and reflected on a sphere. The interferometer beam returns in itself. Each M1 mirror was measured in three different orientations (rotated $120^{\circ}$ ). The test setup is shown in figure 3 .

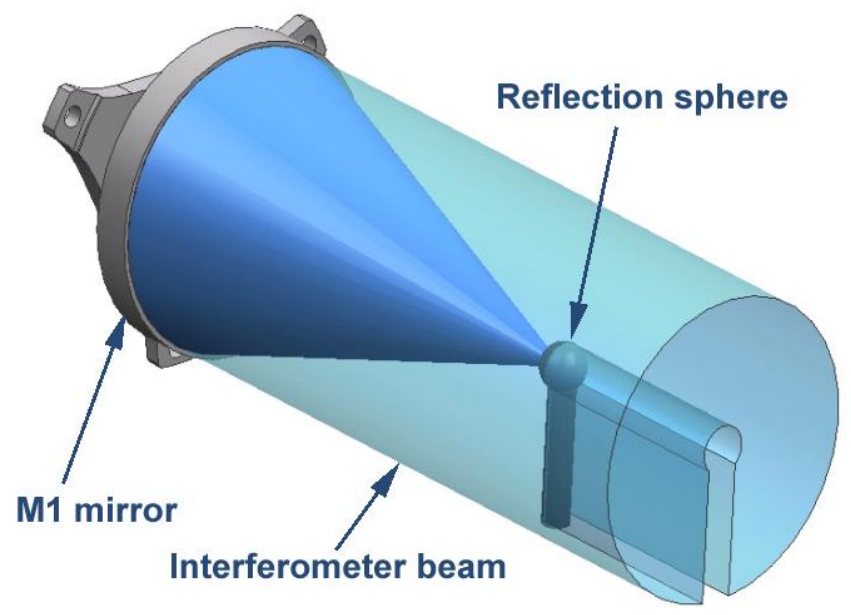

Figure 3 - WFE measurement M1

The flat M2 mirrors were measured directly in front of the WYKO.

The WFE over the entire cats eye shall be better than $30 \mathrm{~nm}$ RMS, including the effects of cool down. The aperture of the incoming science beam of the cats eye is $25 \mathrm{~mm}$. The beam is reflected by the parabolic M1 and focused on the flat M2.

The overall WFE requirement has been translated in a manufacturing WFE requirement for the M1 of $22.4 \mathrm{~nm}$ RMS over the overall diameter of $61.8 \mathrm{~mm}$ and $9 \mathrm{~nm}$ RMS over an aperture of $27 \mathrm{~mm}$. For the M2 the WFE must be less than $22.4 \mathrm{~nm}$ RMS over an aperture of $4 \mathrm{~mm}$.

ESA has not given a requirement for straylight within the scope of this program, but the aim was to achieve a surface roughness as low as possible. The surface roughness of all mirrors was measured with a WYKO profilometer.

A typical WFE interferogram is given in figure 4. An overview of the test results is given in table 1. 


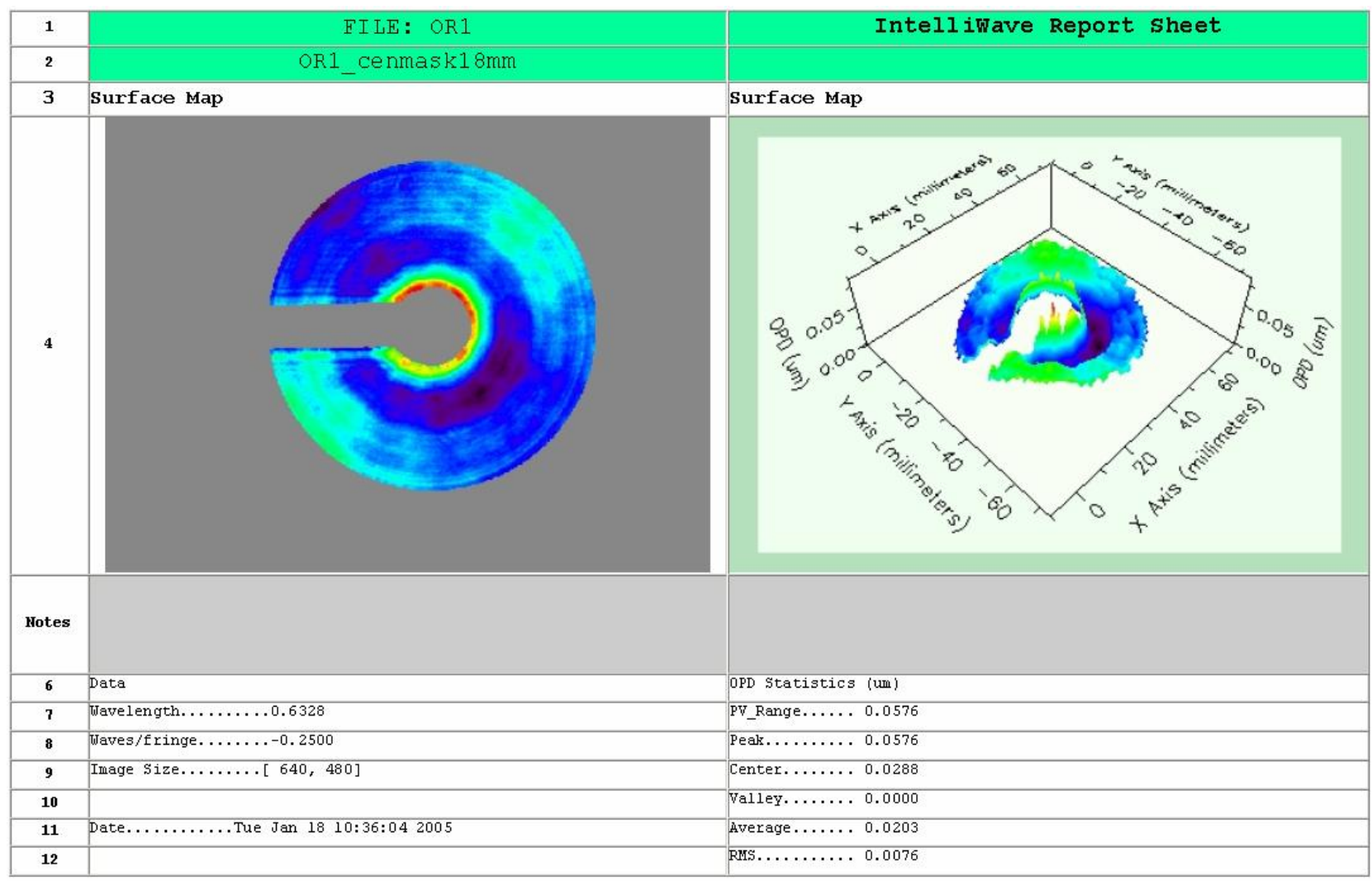

Figure 4 - WFE measurement on M1 s/n 2

WFE and surface roughness measurements in $\mathrm{nm}$ (for M1 averaged over the three orientations)

\begin{tabular}{|c|c|c|c|c|c|}
\hline - WFE measurements M1 & Req. & M1 s/n 2 & M1 s/n 3 & M2 s/n 2 & M2 s/n 3 \\
\hline $\mathrm{P}-\mathrm{V}$ full aperture $(\varnothing=61.8 \mathrm{~mm})$ : & - & 57.8 & 54.2 & & \\
\hline RMS full aperture $(\varnothing=61.8 \mathrm{~mm})$ : & 22.4 & 8.7 & 9.2 & & \\
\hline RMS Sience Beam (Ø $27 \mathrm{~mm}$ ) top: & 9 & 7.5 & $10.9^{*}$ & & \\
\hline RMS Sience Beam (Ø $27 \mathrm{~mm}$ ) bottom: & 9 & 6.4 & 9.1 & & \\
\hline RMS Metrology Beam (Ø $18 \mathrm{~mm})$ : & - & 4.2 & 8.8 & & \\
\hline - WFE measurements M2 & Req. & & & & \\
\hline P-V full aperture $(\varnothing=4 \mathrm{~mm})$ : & - & & & 36.9 & 44.0 \\
\hline RMS full aperture $(\varnothing=4 \mathrm{~mm})$ : & 22.4 & & & 6.9 & 8.7 \\
\hline - $\quad$ RMS Surface Roughness Rq & - & 2.3 & 1.9 & 2.4 & 2.7 \\
\hline
\end{tabular}

* Includes edge effects, the ESA requirement is over an aperture of $25 \mathrm{~mm}$

Table 1 - WFE and surface roughness measurements

An additional set of mirrors, without the full heat treatment program and Alumiplate and Gold coating, was manufactured for the engineering and acceptance test program (M1 s/n 1 and M2 s/n 1). 
After WFE and surface roughness measurements, the mirrors were coated by CSL with a single layer of protective Gold (figure 5). The coating properties of the mirrors were measured at CSL (optical characterisation, microroughness, coating adhesion.

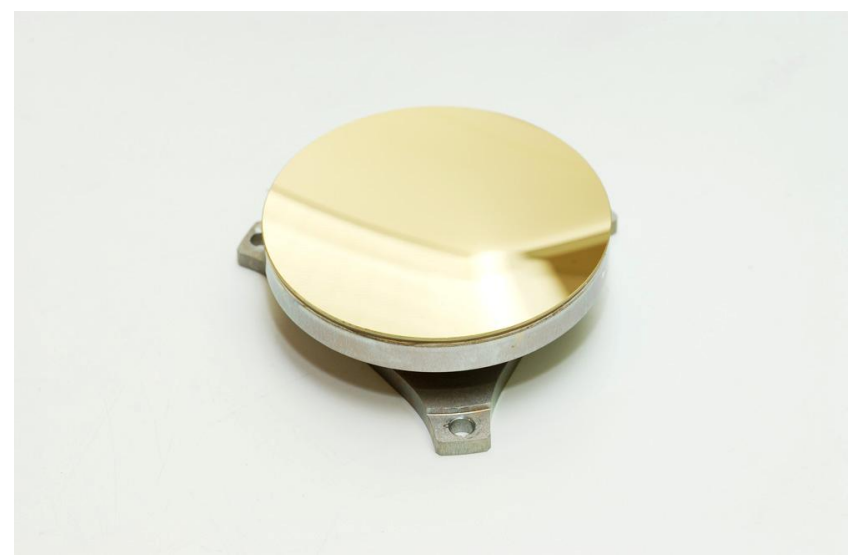

Figure 5 - M1 mirror with protective gold coating

\section{MAGNETIC BEARING MANUFACTURING}

The moving part of the magnetic bearing is an integral part of the cats eye. Therefore both the static and moving part of the magnetic bearing were machined and wire cut from the same lot of material as the mirrors to minimise CTE variations and guarantee the athermal behaviour of the cats eye.

The magnetic bearing was assembled and tested at Micromega in Liege, Belgium, using the set of engineering mirrors which are fully representative in fit form and function. The position of the magnets was adjusted to ensure proper guiding accuracy and minimum power dissipation. The magnetic bearing is equipped with a 1 -g magnetic off loading device, to enable ground testing in horizontal position. The measured power dissipation of the magnetic bearing in the centre position is less than $1 \mathrm{~mW}$. Due to 1 -g loading, the power dissipation in the magnetic bearings at the extreme positions is ca. $20 \mathrm{~mW}$ at $40 \mathrm{~K}$. In 0 -g condition the power dissipation is less than $2 \mathrm{~mW}$.

\section{OPD ACTUATOR MANUFACTURING}

The OPD actuator is a voice coil with a mechanical stroke of $10 \mathrm{~mm}$ (figure 6). The magnet is mounted on the moving part of the ODL, to avoid OPD disturbances and the coil is mounted on the static part of the ODL. Both the voice coil and the amplifier were designed and manufactured by SRON. After manufacturing, the static characteristics of the voice coil and amplifier were measured as function of the position. The voice coil and magnet were subjected to one thermal cycle down to $4 \mathrm{~K}$ in a cryostat at SRON and inspected for cracks and electrical continuity and isolation (figure 7). In order to measure and fine tune the dynamic behaviour of the voice coil actuator, the voice coil was mounted on the ODL at TNO.
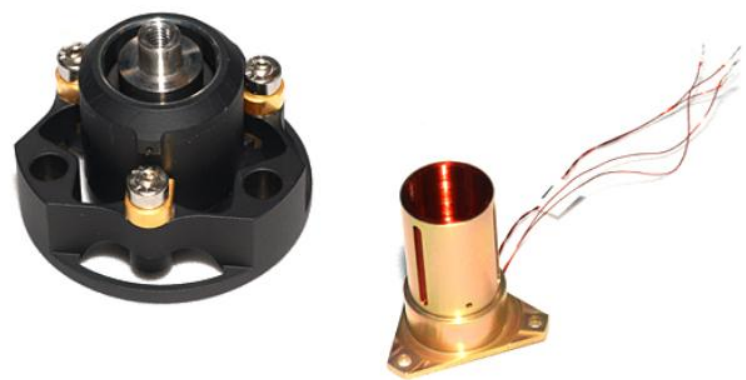

Figure 6 - Voice coil assembly (magnet on the left, coil on the right) 

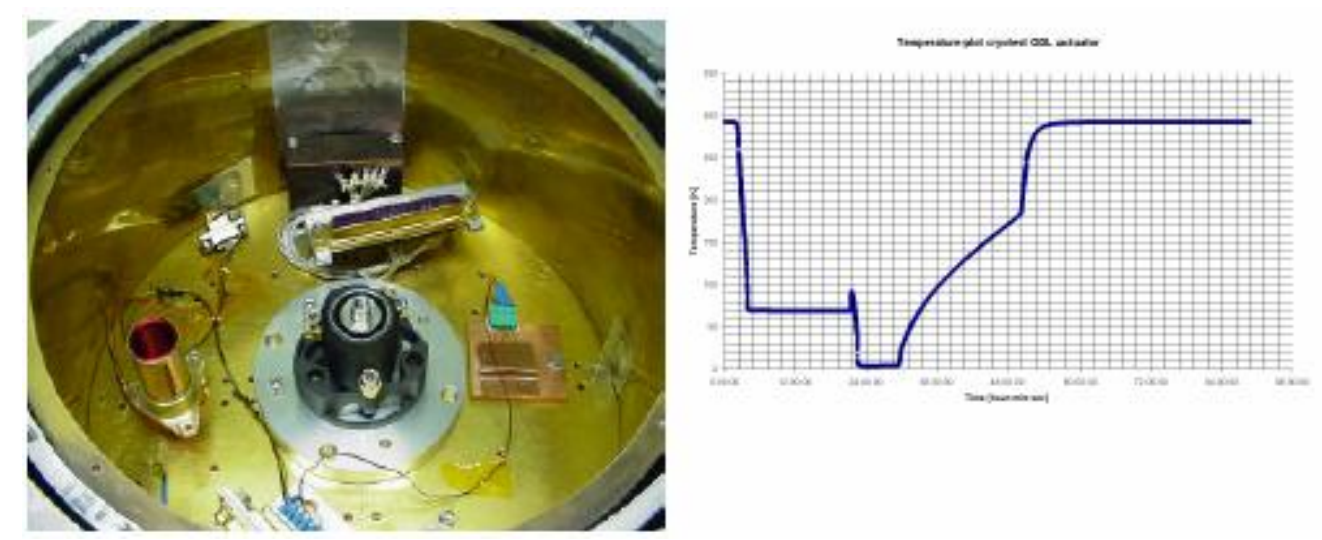

Figure 7 - Thermal cycling of voice coil actuator in SRON cryostat

\section{DELAY LINE ACCEPTANCE TEST PROGRAM}

After completion of component level testing, the OPD controller was fine tuned to obtain a robust OPD stability in the TNO laboratory. The controller uses a laser interferometer with $0.3 \mathrm{~nm}$ resolution for OPD measurement (figure 8). An OPD stability of $0.9 \mathrm{~nm}$ RMS was achieved (against a disturbance spectrum of 2 micron RMS). The PSD plot is given in figure 9. In the DARWIN mission a Fringe Sensor sampling rate between 10 and $100 \mathrm{~Hz}$ will be required to achieve the required $1 \mathrm{~nm}$ RMS OPD stability (depending on the final disturbance spectrum).

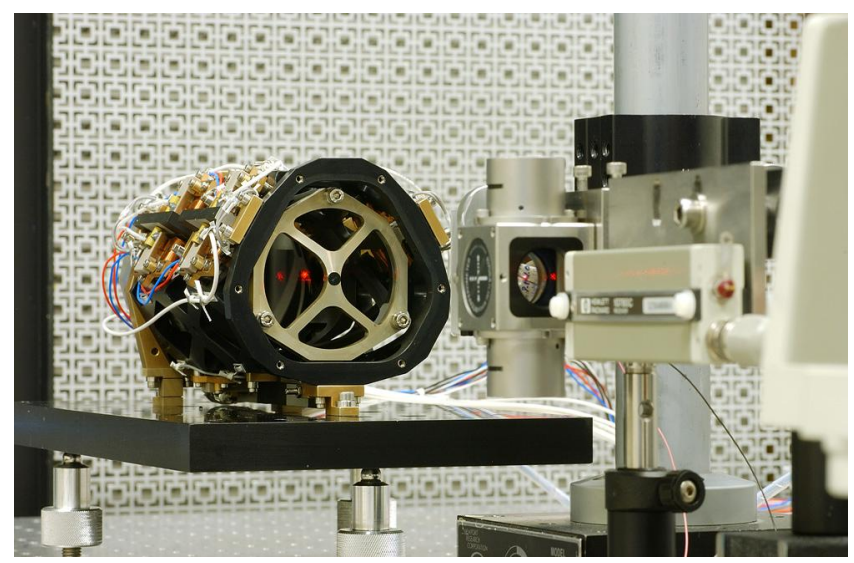

Figure 8. - ODL with laser interferometer 


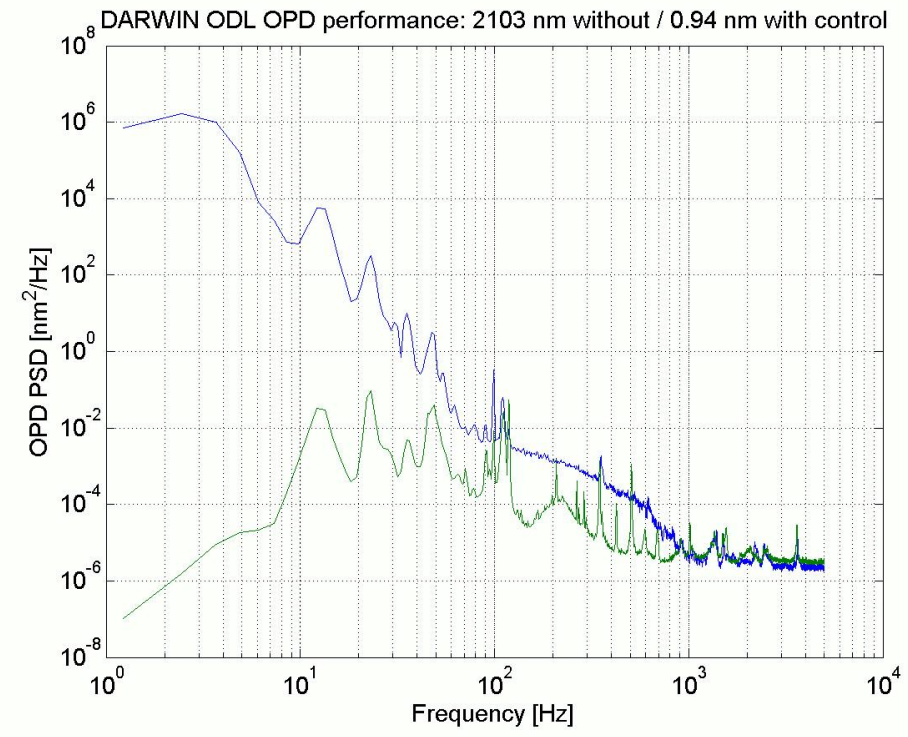

Figure 9 - PSD plot of OPD performance

In order to verify the structural integrity of the design, the complete ODL was subjected to three deep thermal cycles (between +80 and $-185^{\circ} \mathrm{C}$ ) in a Nitrogen gas environment at a facility of Dutch Space in Leiden, The Netherlands (figure 10 and 11). The magnetic bearing was switched on at the first cold phase and operated successfully. During the entire $2^{\text {nd }}$ and $3^{\text {rd }}$ cycle, the magnetic bearing was kept switched on (despite the turbulent gas flow) and operated successfully as well.

After thermal cycling in Nitrogen, the ODL was placed in the thermal vacuum chamber at Dutch Space (figure 12) and subjected to one cool down cycle to $-185^{\circ} \mathrm{C}$ (moving part $-175^{\circ} \mathrm{C}$ ). Both the magnetic bearing and voice coil operated nominally.

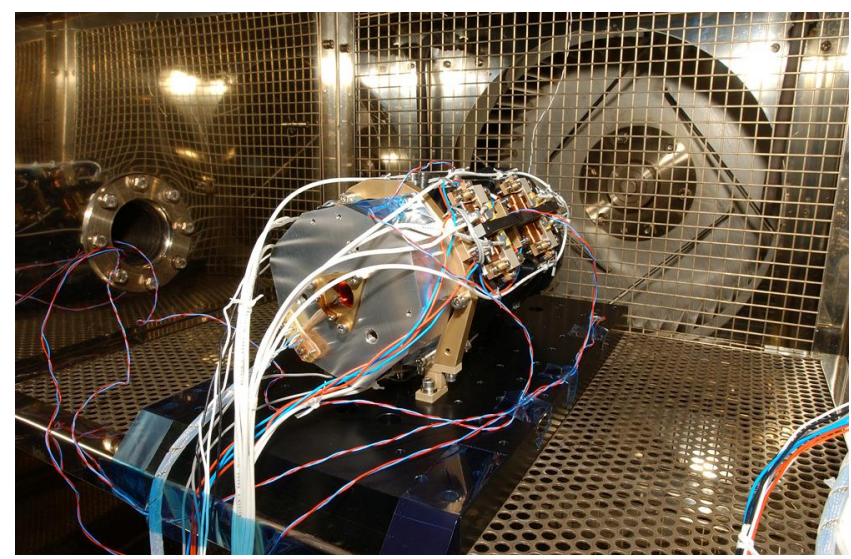

Figure $10-$ ODL thermal cycling at Dutch Space 


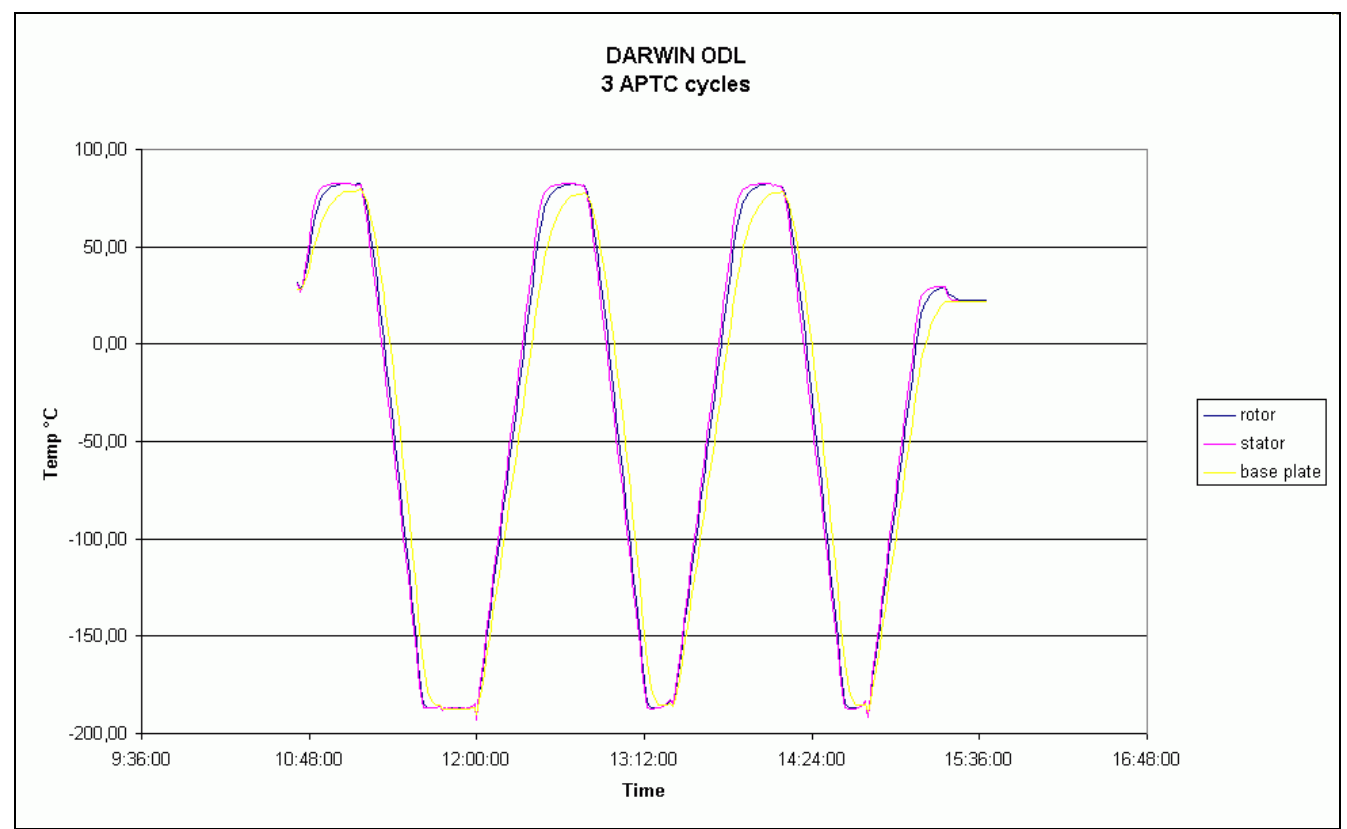

Figure 11 - Thermal cycle profile

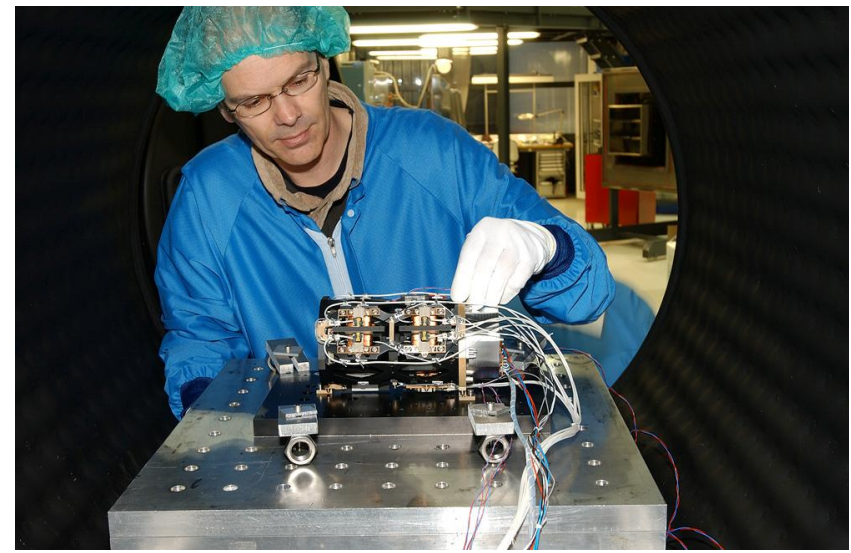

Figure 12 - Thermal vacuum test at Dutch Space

Prior and after the thermal tests, the WFE of the cats eye was measured with a WYKO interferometer (figure 13). The obtained WFE with the engineering mirrors was $<30 \mathrm{~nm}$ RMS. No degradation of the WFE occurred after thermal cycling.

The optical and dynamic performance of the ODL at $40 \mathrm{~K}$ will be measured during the verification program.

Prior to this $40 \mathrm{~K}$ test, which will take place in a thermal vacuum facility at the Centre Spatiale de Liege, a test at $120 \mathrm{~K}$ will be done in the thermal vacuum chamber at Dutch Space, in order to verify the behaviour of the test equipment and delay line. 

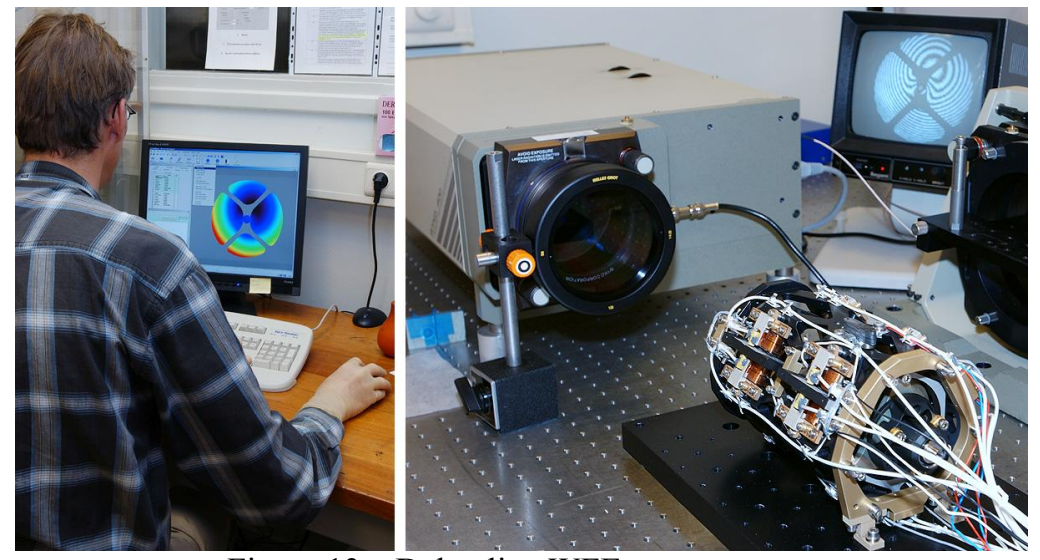

Figure 13 - Delay line WFE measurement

The guiding accuracy of the ODL was measured with an autocollimator on a flat reference mirror at the back of the ODL, mounted on the launch lock interface pin (figure 14). Each magnetic bearing uses two eddy current sensors for position control. The bearing follows the machining accuracy of the target on the moving part (figure 14). The obtained guiding accuracy was $25 \mu \mathrm{rad} \mathrm{P}-\mathrm{V}$, which corresponds to a guiding accuracy of less than $5 \mu \mathrm{m}$ on the magnetic bearing (figure 16). The resulting output beam tilt of the cats eye will be less than $0.1 \mu \mathrm{rad}$ (required $0.24 \mu \mathrm{rad}$ ).

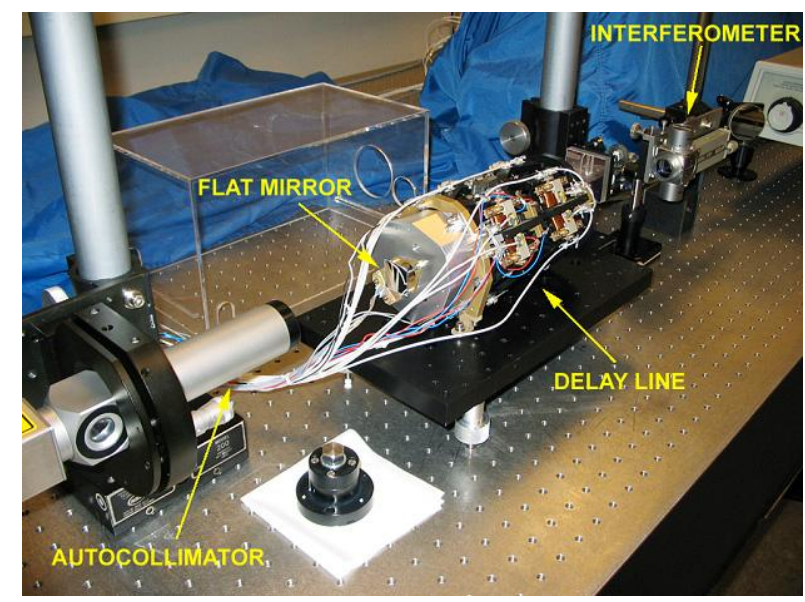

Figure 14 - Tip/tilt measurement

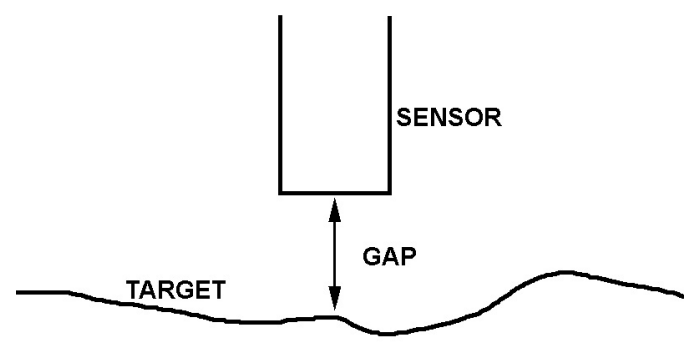

Figure 15 - Eddy current sensor for magnetic bearing gap control 
In order to demonstrate the ultra high accuracy of the guiding ability of the magnetic bearings, the measured gap deviations were placed in a lookup table and the magnetic bearings were driven in active correction mode (in open loop) to correct for these machining tolerances.

The measured guiding accuracy was less than $0.5 \mu \mathrm{rad} \mathrm{P}-\mathrm{V}$, which corresponds to a guiding accuracy of better than $50 \mathrm{~nm}$ (figure 16). The required electrical power dissipation for tilt correction was less than $1 \mathrm{~mW}$.

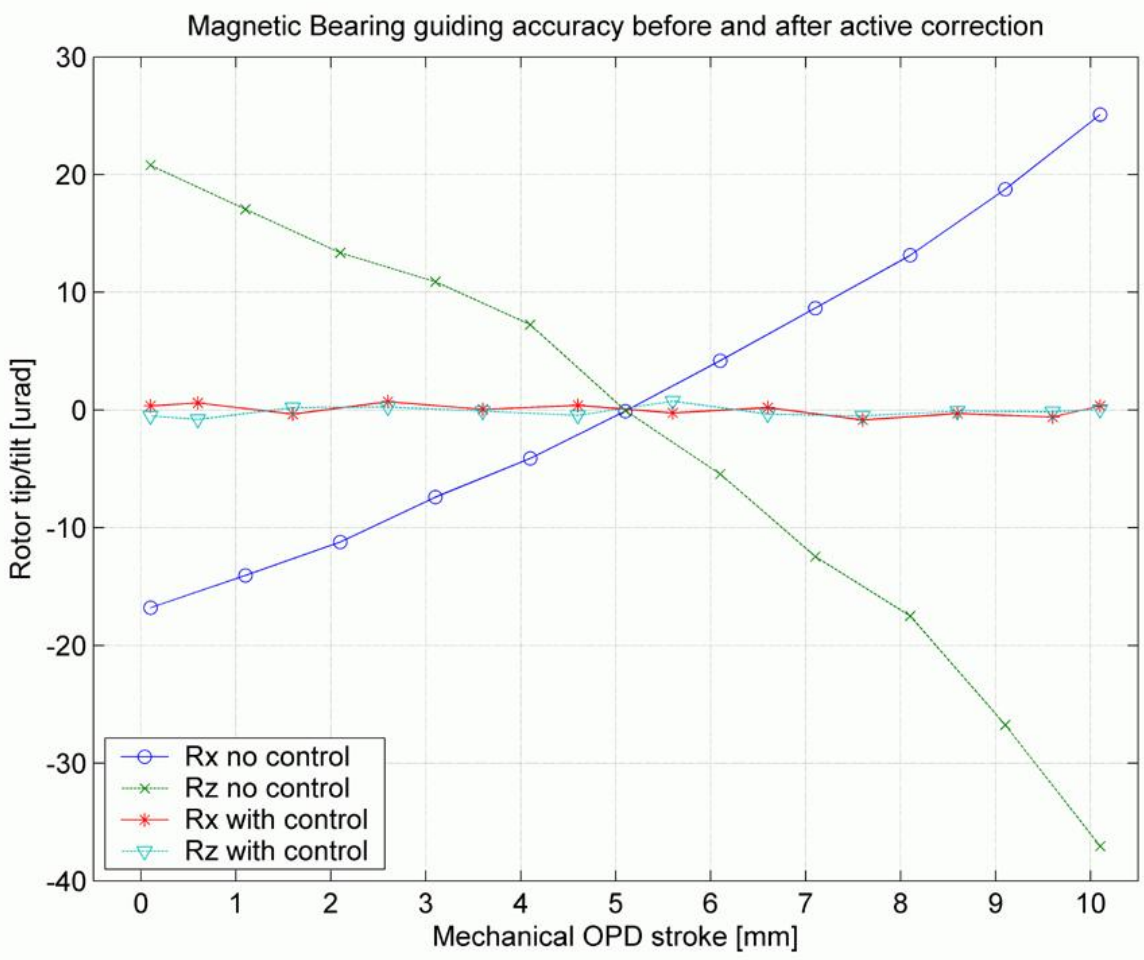

Figure 15 - Magnetic Bearing guiding accuracy in passive and active mode

\section{FUTURE ACTIVITIES}

The acceptance test program on the DARWIN ODL has demonstrated that the design is fully compliant with the ESA requirements. The dynamic and optical performance at $40 \mathrm{~K}$ will be measured during the verification program which will start in the final quarter of 2005.

The current OPD and magnetic bearing controller run on a real time Linux based PC. The power dissipation is not representative for the future DARWIN controller. A DSP based control board dissipates in the order of $15 \mathrm{~W}$ and will not meet the $2.5 \mathrm{~W}$ overall power dissipation requirement.

Currently TNO and SRON are currently developing a low power $(<2 \mathrm{~W})$ FPGA based control board for combined OPD and $\mathrm{MB}$ control.

A launch lock is not part of the current development program, but will be required for a future mission.

After the successful completion of the verification program, TNO is looking for a flight opportunity in a future space interferometer or technology demonstrator mission (please contact ben.braam@tno.nl if you wish to discuss this). TNO is also offering a slightly modified version of this ODL for the ESA GENIE instrument on the ESO VLTI telescopes.

This delay technology can also be used in other ground based astronomical instruments [4]. 


\section{AKNOWLEDGEMENTS}

TNO would like to thank Josep Maria Perdigues Armengol (ESA-ESTEC), Joost Carpay (NIVR) and Gustavo Vallejo (Alumiplate) for their support during the development of the delay line.

\section{REFERENCES}

[1] DARWIN-The InfraRed Space Interferometer, Concept and Feasibility Study Report", Version 1.2 ESASCI(2002)12, April 2002

[2] Statement of Work, Optical Delay Lines, Programme Reference: TRP, ID-OP-12 TOS-MMO/2002/276, issue 2.0, 6 March 2003.+ clarifications 1, 2 and 3

[3] T.C. van den Dool et al, 'The design of a breadboard Cryogenic Optical Delay Line for DARWIN', SPIE Conference Astronomical Telescopes and Instrumentation Vol. 5495-40, Glasgow, June 2004

[4] T.C. van den Dool et al, 'Advanced optical delay line demonstrator', SPIE Conference Astronomical Telescopes and Instrumentation Vol. 5495-41, Glasgow, June 2004

[5] B. Snijders et al., "Free-beam delay line for a multi-aperture optical space interferometer stabilized on a guide star", SPIE Vol. 2209, 1994

[6] B.C.Braam et al., "Kinematic six-ball guide for long stroke optical delay line", Proc. Sixth European Space Mechanisms \& Tribology Symposium, Zurich (Switzerland), 1995, ESA SP-374, August 1995

[7] M.R. Swain, P.R. Lawson, J.D. Moore, and D. Jennings, 'Cryogenic delay line for far-IR interferometry in space', 36th International Astrophysical Colloquium, 2-5 July 2001, Liege, Belgium.

[8] 'Active Vibration Control for an Optical Delay Line', N.J. Doelman, T.C. van den Dool. Proc. of Active 2002, pp.887-898

[9] Improved cryogenic aluminum mirrors, D. Vukobratovich, K. Don, R. E. Sumner, National Optical Astronomy Observatories, SPIE Vol. 3435-02 (1999)

[10] Diamonds turn infrared mirrors smooth, D. Vukobratovich, K. Don, R. E. Sumner, National Optical Astronomy Observatories, Optoelectronics World, October 1998

[11] MABE, Fine Precision Mechanism Based on Magnetic Bearing Technology, Micromega Dynamics - Executive Summary (ESA Contract No 13676/99/NL/PA) 